\title{
Microtubule-associated deacetylase HDAC6 promotes angiogenesis by regulating cell migration in an EB1-dependent manner
}

\author{
Dengwen $\mathrm{Li}^{1 *}$, Songbo $\mathrm{Xie}^{1 *}$, Yuan Ren ${ }^{1 *}$, Lihong Huo ${ }^{1}$, Jinmin Gao ${ }^{1}$, Dandan Cui ${ }^{1}$, Min Liu ${ }^{2}$, Jun Zhou ${ }^{1 凶}$ \\ ${ }^{1}$ Department of Genetics and Cell Biology, Tianjin Key Laboratory of Protein Science, College of Life Sciences, Nankai \\ University, Tianjin 300071, China \\ 2 Department of Biochemistry, Tianjin Key Laboratory of Cellular and Molecular Immunology, Basic Medical College, Tianjin \\ Medical University, Tianjin 300071, China \\ $\bowtie$ Correspondence: junzhou@nankai.edu.cn
}

Received January 22, 2011 Accepted February 7, 2011

\begin{abstract}
Angiogenesis, a process by which the preexisting blood vasculature gives rise to new capillary vessels, is associated with a variety of physiologic and pathologic conditions. However, the molecular mechanism underlying this important process remains poorly understood. Here we show that histone deacetylase 6 (HDAC6), a microtubule-associated enzyme critical for cell motility, contributes to angiogenesis by regulating the polarization and migration of vascular endothelial cells. Inhibition of HDAC6 activity impairs the formation of new blood vessels in chick embryos and in angioreactors implanted in mice. The requirement for HDAC6 in angiogenesis is corroborated in vitro by analysis of endothelial tube formation and capillary sprouting. Our data further show that HDAC6 stimulates membrane ruffling at the leading edge to promote cell polarization. In addition, microtubule end binding protein 1 (EB1) is important for HDAC6 to exert its activity towards the migration of endothelial cells and generation of capillary-like structures. These results thus identify HDAC6 as a novel player in the angiogenic process and offer novel insights into the molecular mechanism governing endothelial cell migration and angiogenesis.
\end{abstract}

KEYWORDS angiogenesis, histone deacetylase 6 (HDAC6), cell migration, cell polarization, microtubule end binding protein 1 (EB1)

\section{INTRODUCTION}

The blood vessel network arises through two distinct processes, vasculogenesis and angiogenesis. Vasculogenesis refers to the de novo generation of blood vessels from vascular progenitor cells during embryogenesis, whereas angiogenesis refers to the formation of new capillaries via remodeling of or expansion from preexisting blood vessels (Semenza, 2007). Angiogenesis is a complex event that requires an exquisite interplay between different cell types (Folkman, 2007). The angiogenic process is critical for the maintenance of oxygen homeostasis and is tightly regulated in normal physiologic settings (e.g., during wound healing or female menstrual cycle). Aberrant (insufficient or exaggerated) angiogenesis is associated with a variety of pathologic conditions such as ischemia, sclerodermia, psoriasis, and malignancy (Folkman, 2007).

Vascular endothelial cell migration plays an essential role in the angiogenic process, from the initial opening of blood vessels to the final closure of a functional vasculature (Lamalice et al., 2007). Endothelial cells establish their polarity in response to migratory cues by forming gradients of signaling molecules and reorganizing the organelles (Lamalice et al., 2007; Bornens, 2008; Li and Gundersen, 2008). For example, the centrosome is randomly localized in quiescent endothelial cells and reorients towards the leading edge in the presence of migratory stimuli (Bornens, 2008). Efficient cell migration also relies on dynamic rearrangement of the cytoskeleton (Rodriguez et al., 2003). Especially, a number of signaling molecules can associate with cytoske-

\footnotetext{
*These authors contributed equally to this work.
} 
letal components and modulate cytoskeletal dynamics, thereby promoting cell polarization and migration.

Histone deacetylases (HDACs) are a family of enzymes that have been extensively studied for their roles in regulating chromatin dynamics and gene transcription (Haberland et al., 2009). A number of HDAC inhibitors are in clinical development as anticancer agents (Xu et al., 2007), and one of them, suberoylanilide hydroxamic acid, has recently been approved for the treatment of advanced cutaneous $T$ cell lymphoma (Marks, 2007). While most of the HDACs are localized in the nucleus, HDAC6 is a cytoplasmic protein associated with the cytoskeleton and involved in cytoskeleton-related activities (Boyault et al., 2007; Valenzuela-Fernández et al., 2008). There is a growing body of evidence showing that HDAC6 functions at the intersection of the cytoskeleton and cell signaling to regulate cell motility, cell adhesion, and cell-cell interactions (Boyault et al., 2007; Valenzuela-Fernández et al., 2008). These findings suggest that this deacetylase might have a role in angiogenesis, which is critically dependent on the aforementioned cellular activities (Lamalice et al., 2007). In the present study, we report that HDAC6 promotes angiogenesis by regulating the migration of vascular endothelial cells through the EB1 axis.

\section{RESULTS}

\section{HDAC6 activity is critical for angiogenesis in chick embryos}

To test whether HDAC6 plays a role in the angiogenic process, we performed angiogenesis assays using chick embryos. Filter paper soaked with tubacin, a specific inhibitor of HDAC6 (Haggarty et al., 2003), or the vehicle DMSO was placed on the vitelline membrane of a 4-day-old chick embryo cultured ex ovo (Fig. 1A), and capillaries underneath the filter paper were examined 3 days later (Fig. 1B). We found that tubacin dramatically inhibited the growth of blood vessels in the vitelline membrane (Fig. $1 \mathrm{~B}$ and $1 \mathrm{C}$ ). To eliminate the interference of preexisting blood vessels, matrigel with tubacin or DMSO was polymerized around two layers of nylon mesh and placed on the chorioallantoic membrane of a 10-day-old chick embryo (Fig. 1D). Blood vessel growth into matrigel/nylon mesh was examined 3 days later. As shown in Fig. $1 \mathrm{E}$ and $1 \mathrm{~F}$, inhibition of HDAC6 activity by tubacin reduced the formation of new blood vessels in matrigel/nylon mesh. These data indicate the requirement for HDAC6 activity in embryonic angiogenesis.

\section{HDAC6 mediates angiogenic response in mice}

The involvement of HDAC6 in embryonic angiogenesis prompted us to study its potential role in postnatal angiogenesis. We first examined the expression pattern of HDAC6 in mouse liver, small intestine, adrenal gland, and esophagus tissues. By immunostaining with antibodies against HDAC6 and the endothelial cell marker CD31, we found that HDAC6 was ubiquitously expressed in these tissues and was partly localized in the vascular endothelium (Fig. 2A). We then performed directed angiogenesis assay using 6- to 8-weekold mice to investigate the role of HDAC6 in postnatal angiogenesis. Semiclosed angioreactors containing matrigel were implanted subcutaneously into the dorsal flank of mice, and the angioreactors were removed and photographed 11 days later for evaluation of angiogenic response (Fig. 2B). As expected, heparin/FGF2 stimulated blood vessel growth into the angioreactors (Fig. $2 \mathrm{C}$ and 2E). Strikingly, the angiogenic response induced by heparin/FGF2 was significantly blocked by addition of tubacin (Fig. $2 \mathrm{D}$ and $2 \mathrm{E}$ ).

Frozen sections of the vessel-containing matrigel were then stained with anti-CD31 antibody and DAPI to examine the vessel architecture in angioreactors. As shown in Fig. $2 \mathrm{~F}$, blood vessels were observed in the control group but undetectable in the tubacin-treated group. To further quantify the angiogenic response, cell pellets were recovered from the angioreactors and stained with FITC-lectin. We found that the fluorescence intensity of cells recovered from tubacin-treated angioreactors was significantly decreased compared with the control group (Fig. 2G). Collectively, these results demonstrate that HDAC6 activity is crucial for postnatal angiogenesis.

\section{HDAC6 promotes endothelial tube formation and capillary sprouting}

To better characterize the role of HDAC6 in angiogenesis, we performed endothelial tube formation and capillary sprouting assays using HUVECs. Tube-like structures were discernable $4 \mathrm{~h}$ after plating cells onto matrigel, and the structures were more distinct $24 \mathrm{~h}$ after plating. Treatment of cells with tubacin significantly impaired tube formation as compared to the control group (Fig. 3A). Measurement of the cumulative tube length revealed that tubacin reduced tube formation from HUVECs by $67 \%, 52 \%$ and $56 \%$, respectively, 4,24 and $36 \mathrm{~h}$ after plating (Fig. 3B). Similarly, knockdown of HDAC6 expression using two specific siRNAs remarkably impaired the ability of HUVECs to form tube-like structures (Fig. 3C-E).

We then used collagen-based 3-dimensional angiogenesis assay to study the effect of HDAC6 on capillary sprouting. HUVECs were suspended in culture medium containing carboxymethylcellulose and seeded onto round-bottom plates to form spheroids. The spheroids were then embedded into collagen gels, and culture medium was added on top of the gel to allow for capillary-like sprout formation. We found that treatment of the spheroids with tubacin decreased their sprouting ability by $71 \%$ (Fig. $3 \mathrm{~F}$ and $3 \mathrm{G}$ ). In addition, the two HDAC6 siRNAs inhibited capillary spouting from endothelial spheroids by $73 \%$ and $78 \%$, respectively (Fig. $3 \mathrm{H}$ and $3 \mathrm{l}$ ). These data provide evidence that HDAC6 promotes angiogenesis through its actions in endothelial cells. 


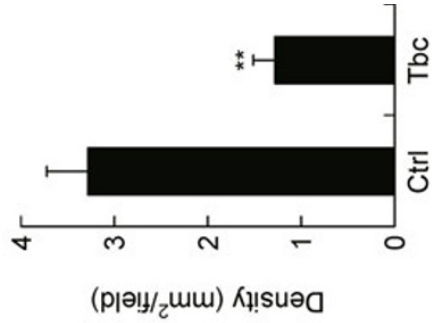

u

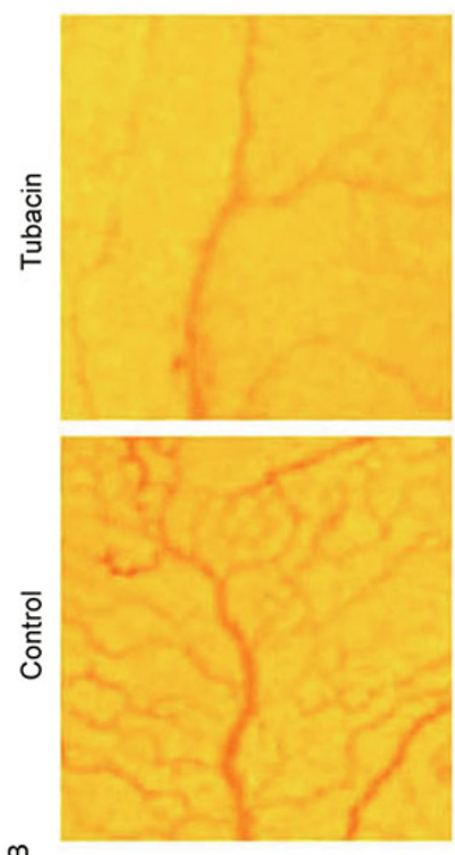

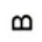

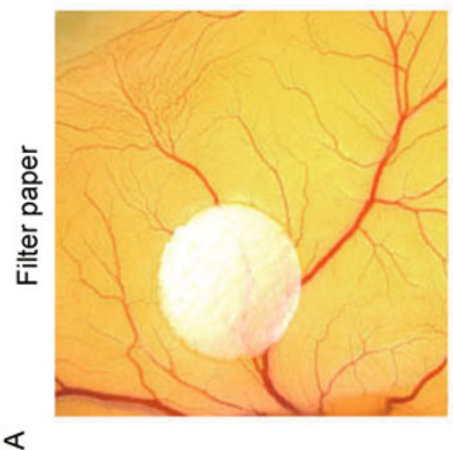

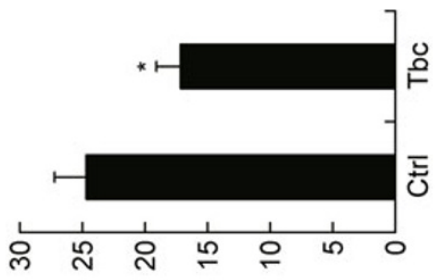

(\%) sp!̣6 ә әм!!!sod

แ
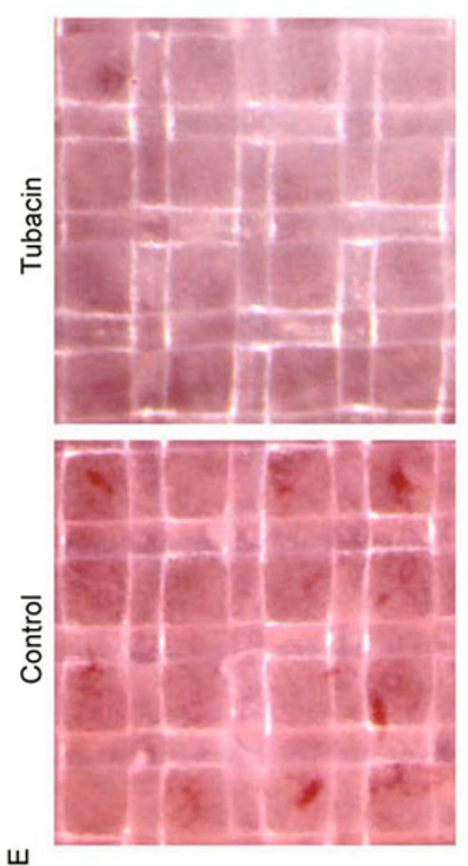

ш

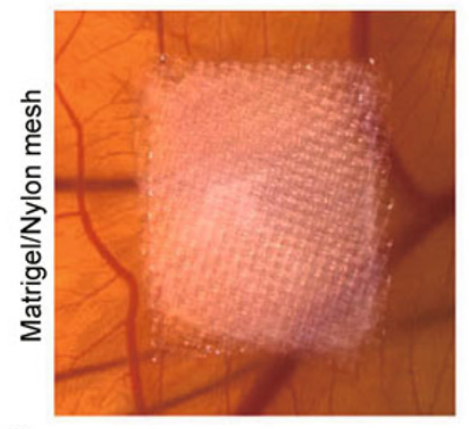

可 0 웡

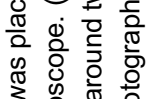

흐을 웜

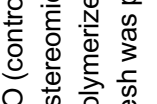

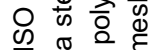

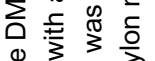

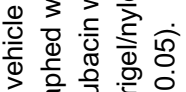

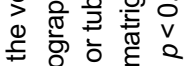

ㅎํㅇㅇㅇㅇㅇㅇ

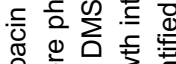

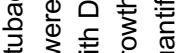

훙 항

啫

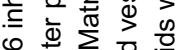

记 난

잉응

政

焉 0 过

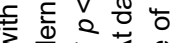

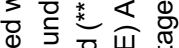

舫

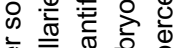

बू.

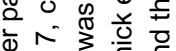

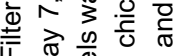

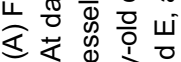

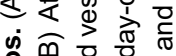

कำ

है

बे

웡

ठ

들 छ

क O

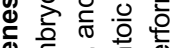

के

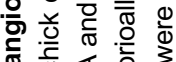

50 品

훈 믕 $\frac{0}{0} 0$

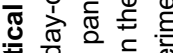

군

is

ㄴㅎㅇ $\frac{0}{0}$

突

: है

U

오

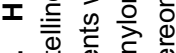

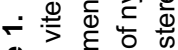

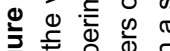

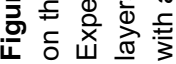




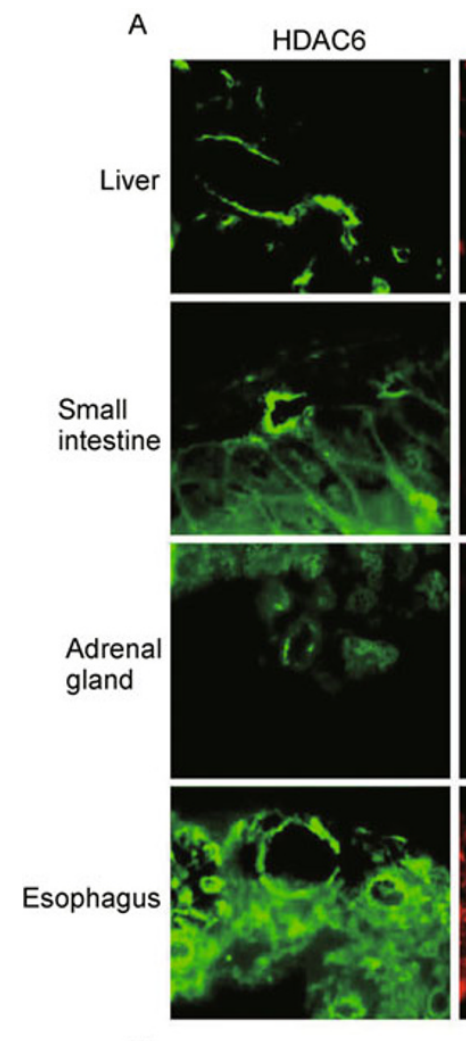

$\mathrm{E}$

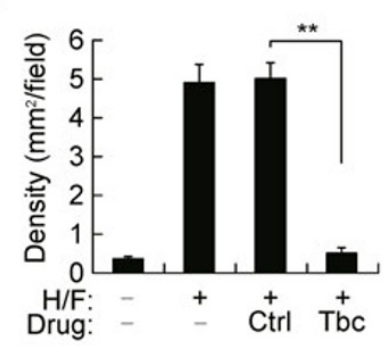

B
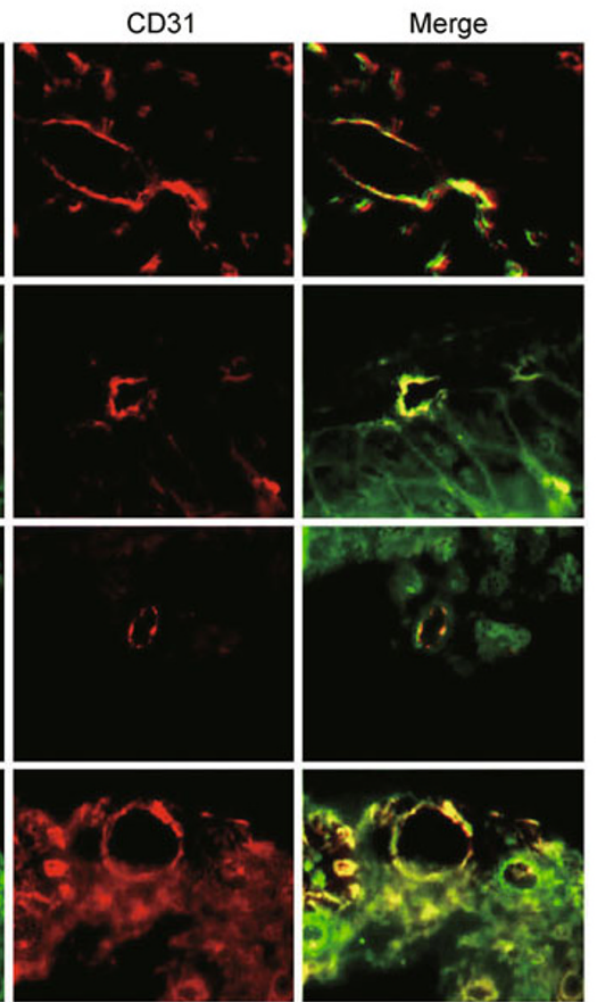

$\mathrm{F}$
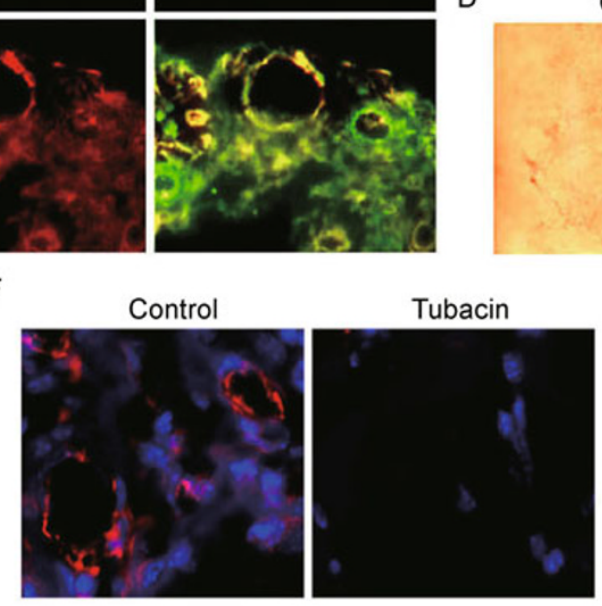

G

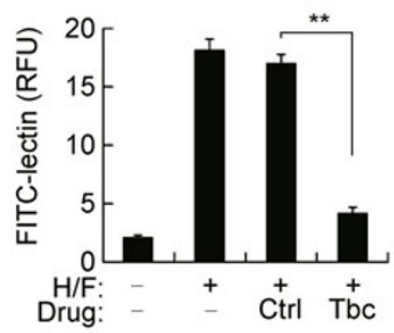

Figure 2. Inhibition of HDAC6 activity impairs blood vessel growth into the angioreactors implanted in mice. (A) Immunolocalization of HDAC6 in mouse tissues. Frozen sections of liver, small intestine, adrenal gland, and esophagus tissues were stained with antibodies against HDAC6 and CD31. The localization of HDAC6 in the vascular endothelium was then examined with a fluorescence microscope. (B) Diagram of directed in vivo angiogenesis assay. Semiclosed angioreactors were implanted subcutaneously into the dorsal flank of athymic nude mice for 11 days, and vascular growth into the angioreactors was photographed (panels $C$ and D). In panel C, angioreactors were filled with matrigel in the absence or presence of heparin/FGF2. In panel D, angioreactors were filled with matrigel in the presence of heparin/FGF2 and DMSO or tubacin. (E) Quantification of the density of blood vessels in the angioreactors $\left({ }^{* *} p<0.01\right)$. (F) Frozen sections of the vessel-containing matrigel in angioreactors were stained with antiCD31 antibody (red) and the nuclear dye DAPI (blue). (G) Cell pellets extracted from the vessel-containing angioreactors were stained with FITC-lectin, and the angiogenic response was reflected by the fluorescence intensity $\left({ }^{* *} p<0.01\right)$. RFU, relative fluorescence unit.

\section{HDAC6 mediates vascular endothelial cell migration}

Given the importance of vascular endothelial cell migration in angiogenesis, we analyzed the effect of HDAC6 on the migratory properties of HUVECs. By in vitro wound healing assay, we found that inhibition of HDAC6 activity by tubacin significantly inhibited the migration of HUVECs, as evidenced by compromised wound closure (Fig. 4A and 4B). Similarly, knockdown of HDAC6 expression by the two different siRNAs inhibited wound closure by $62 \%$ and $65 \%$, respectively (Fig. 4C and 4D). To further investigate the action of HDAC6 in endothelial cell migration, the extent of wound 
A
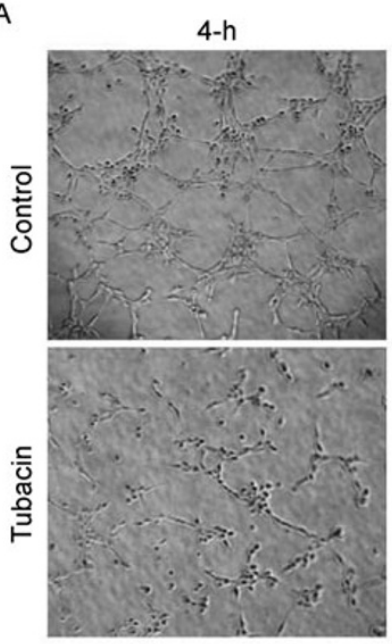

D

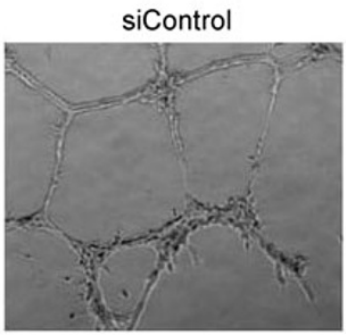

F

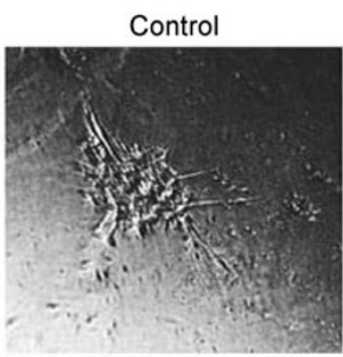

$\mathrm{H}$

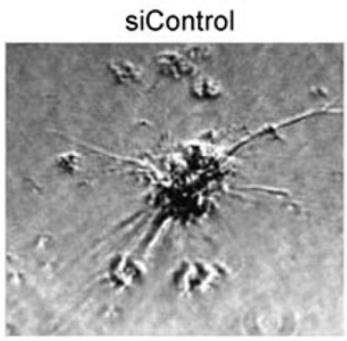

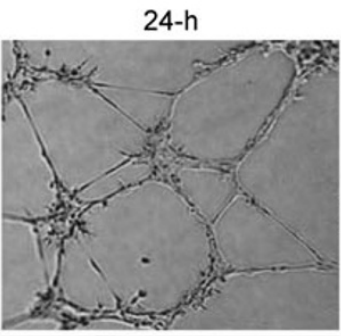
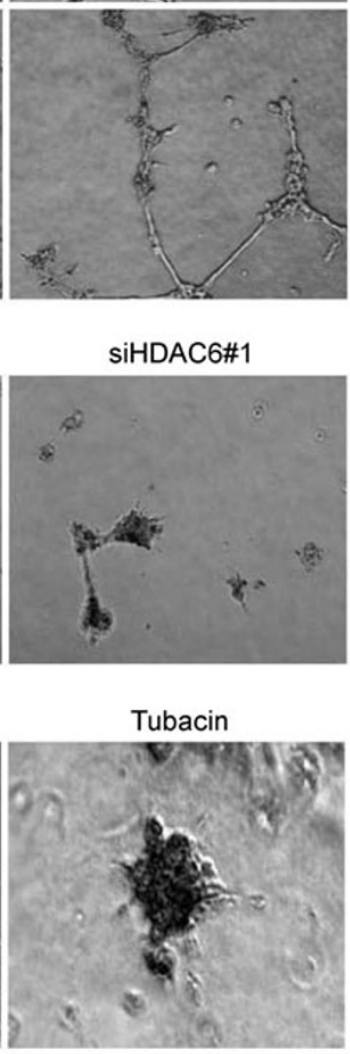

siHDAC6\#1

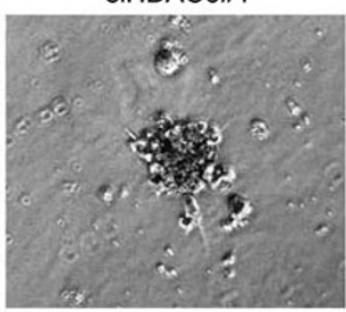

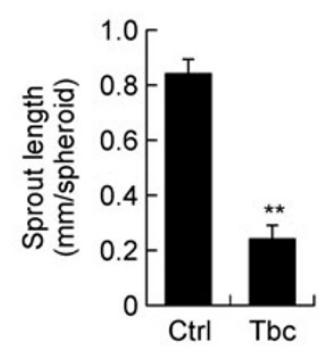
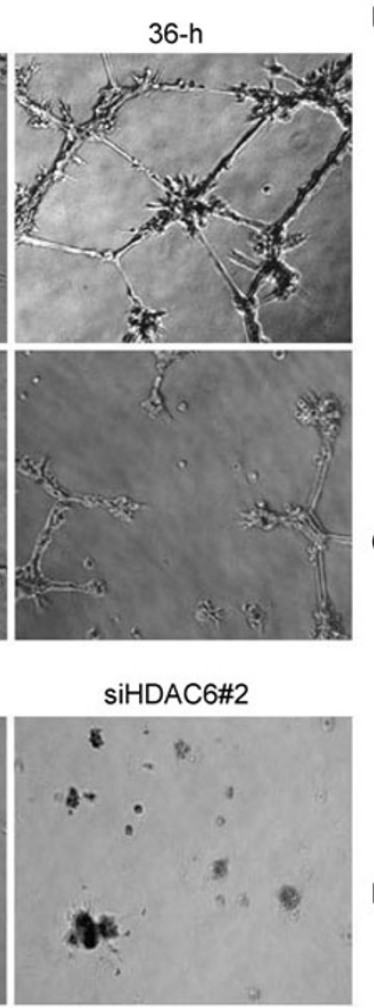

G

siHDAC6\#2

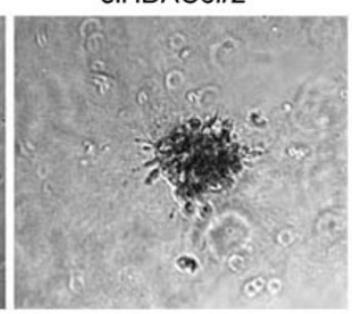

E
B

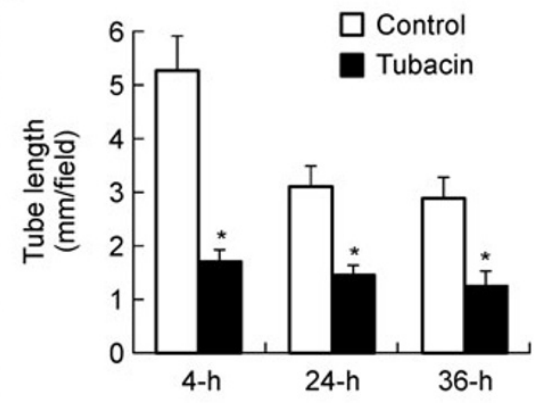

C
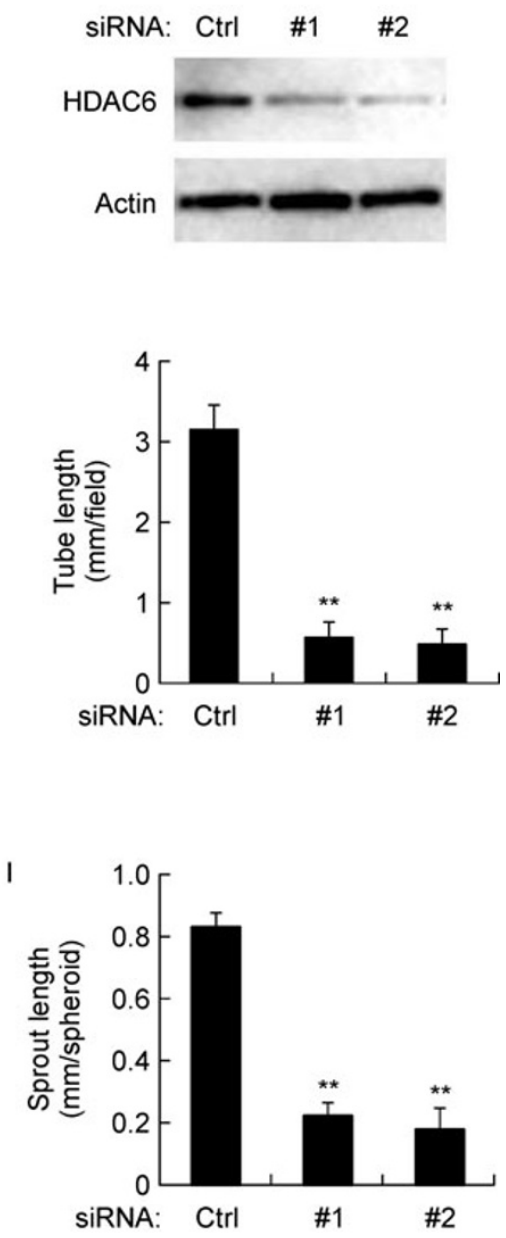

Figure 3. HDAC6 is required for endothelial tube formation and capillary sprouting. (A) HUVECs were plated onto matrigel and treated with DMSO or tubacin for 4, 24, or $36 \mathrm{~h}$, and the formation of endothelial tubes was examined. (B) Experiments were performed as in panel $A$, and cumulative tube length was quantified $\left({ }^{*} p<0.05\right.$ vs control). (C) HUVECs were transfected with HDAC6 or control siRNAs, and the expression of HDAC6 and actin was examined by immunoblotting. (D) HUVECs transfected with HDAC6 or control siRNAs were plated onto matrigel, and photographs were taken $24 \mathrm{~h}$ later. $(E)$ Experiments were performed as in panel $D$, and cumulative tube length was quantified (** $p<0.01$ vs control). (F) Spheroids generated from HUVECs were treated with DMSO or tubacin, and capillary-like sprout formation was then examined. $(G)$ Experiments were performed as in panel $F$, and cumulative sprout length was quantified $\left({ }^{* *} p<0.01\right.$ vs control). ( $\mathrm{H}$ and $\left.\mathrm{I}\right)$ Capillary sprouting from spheroids generated from HUVECs transfected with HDAC6 or control siRNAs (** $p<0.01$ vs control). 

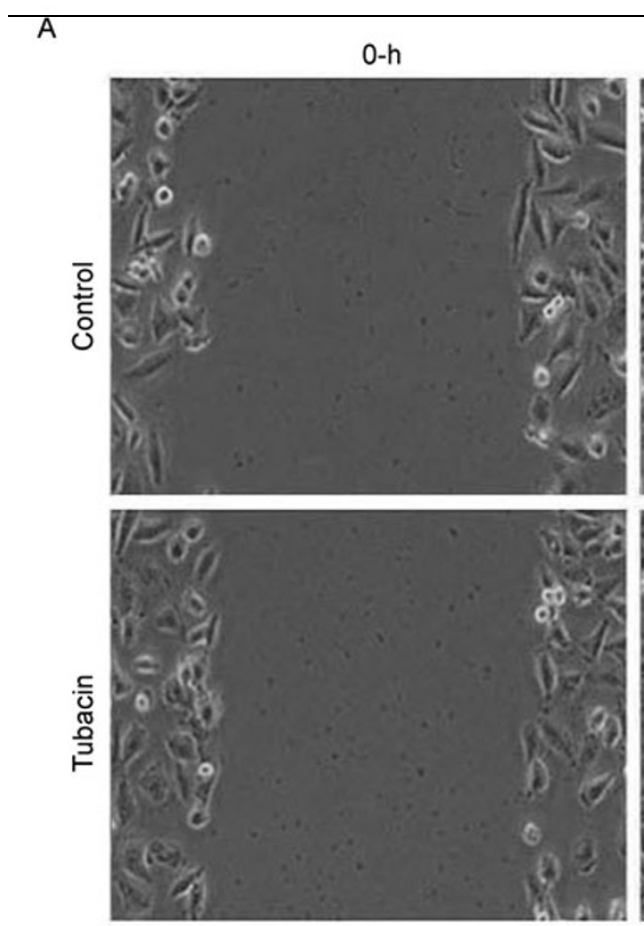

C

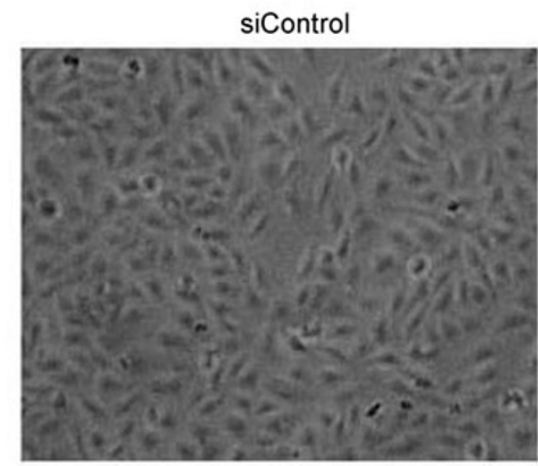

E

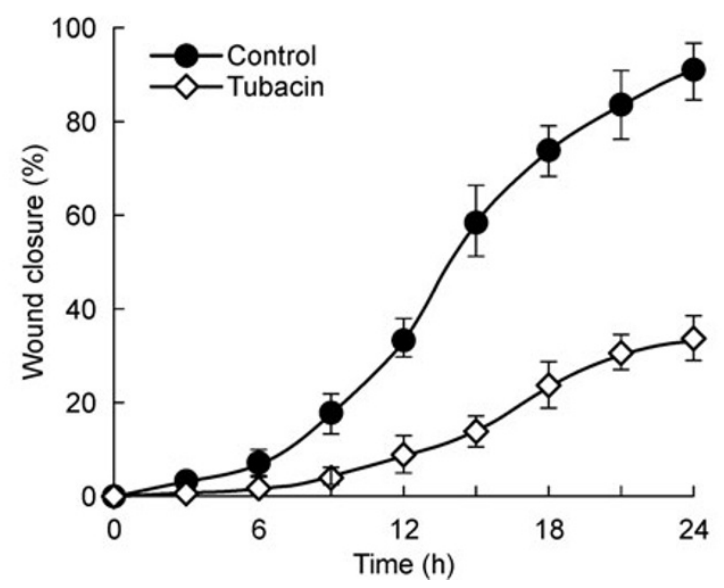

24-h
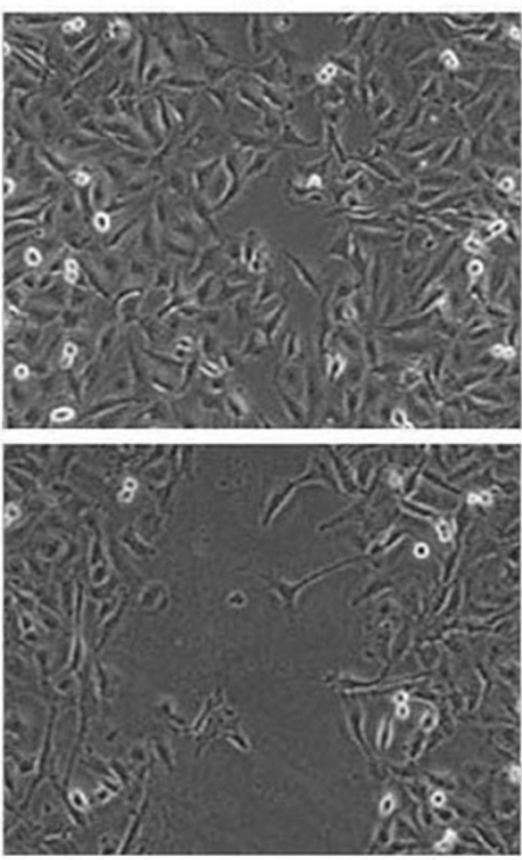

siHDAC6\#1

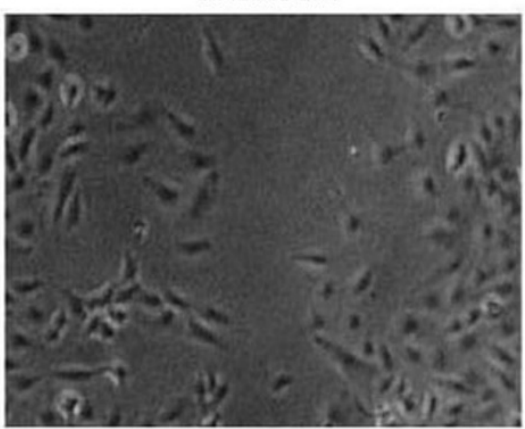

B

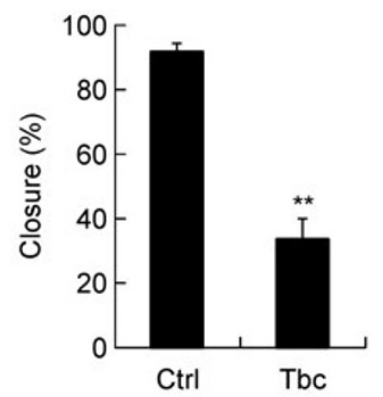

D

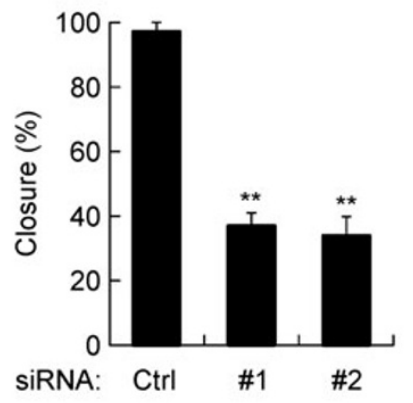

siHDAC6\#2

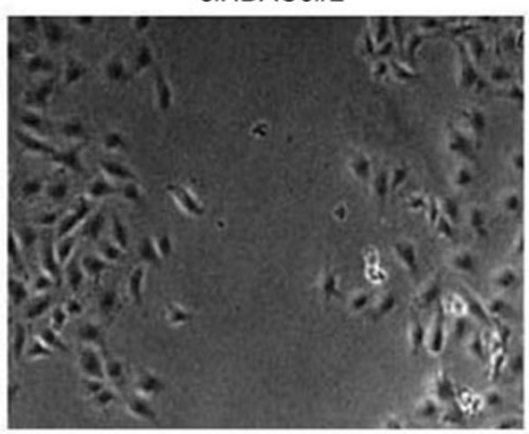

F

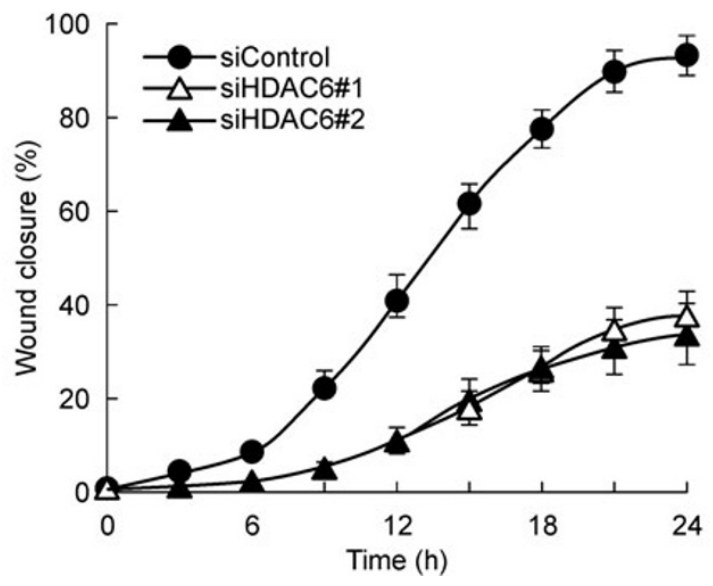

Figure 4. HDAC6 mediates vascular endothelial cell migration. (A) HUVECs were treated with DMSO or tubacin and scratched, and wound margins were photographed 0 or $24 \mathrm{~h}$ later. (B) Experiments were performed as in panel $\mathrm{A}$, and the extent of wound closure was quantified by measuring the wound area compared with the initial wound area $\left({ }^{* *} p<0.01\right.$ vs control). (C) HUVECs transfected with control or HDAC6 siRNAs were scratched, and wound margins were photographed $24 \mathrm{~h}$ later. (D) Experiments were performed as in panel $\mathrm{C}$, and the extent of wound closure was quantified ( ${ }^{* *} p<0.01 \mathrm{vs}$ control). ( $\mathrm{E}$ and $\mathrm{F}$ ) Experiments were performed as in panels $\mathrm{A}$ and $\mathrm{C}$, respectively, and the extent of wound closure was examined at different time points. 
closure was examined at different time points after scratching. As shown in Fig. 4E and 4F, treatment with tubacin or transfection with HDAC6 siRNAs impaired the migration of HUVECs in a time-dependent manner. These results thus indicate that HDAC6 plays an important role in the migration of vascular endothelial cells.

\section{HDAC6 stimulates membrane ruffling and cell polariza- tion}

Cell migration is known to involve dynamic membrane ruffling at the leading edge of cells (Lamalice et al., 2007; Li and Gundersen, 2008). To investigate whether HDAC6 promotes endothelial cell migration through effects on membrane ruffling, HUVECs were transfected with pEGFPC1 and scratched, and the fluorescence of green fluorescence protein (GFP) at the leading edge was recorded at 20-second intervals (Fig. 5A). Three-dimensional surface plots depicting the dynamic changes in GFP fluorescence were then generated from the sequential images as a measure of membrane ruffle dynamics. We found that inhibition of HDAC6 activity by tubacin dramatically reduced the changes in GFP fluorescence at the leading edge of migrating HUVECs, indicating weakened membrane ruffling (Fig. 5B).

The involvement of HDAC6 in modulation of membrane ruffle dynamics suggests that it might affect endothelial cell polarization, of which the formation of membrane ruffles is an essential component. To address this possibility, HUVECs were treated with DMSO or tubacin and scratched, and then immunostained to visualize microtubules, centrosomes, and nuclei at the wound margin. We found that at the initial time point of scratching $(0 \mathrm{~h})$, only $33 \%$ of cells at the wound margin had a polarized morphology with centrosomes localized between the nuclei and the leading edge (Fig. 5C and $5 \mathrm{D})$. The percentage of polarized cells at the wound margin was increased to $91 \%$ during migration ( $3 \mathrm{~h}$ after scratching). In contrast, cells treated with tubacin largely failed to establish the polarity, with centrosomes randomly localized (Fig. 5C and 5D). We also found that knockdown of HDAC6 expression by siRNAs significantly inhibited the polarization of HUVECs (Fig. 5E and 5F). Collectively, these findings demonstrate a critical requirement for HDAC6 in membrane ruffling and polarization of vascular endothelial cells.

\section{HDAC6 promotes cell migration and angiogenesis through the EB1 axis}

We then sought to gain more mechanistic insights into the actions of HDAC6 in endothelial cell migration and angiogenesis. The microtubule-tip associated protein EB1 plays an essential role in cell polarization and migration (Lansbergen and Akhmanova, 2006), and has recently been shown to interact with HDAC6 and implicated in HDAC6-mediated activities (Zilberman et al., 2009). We therefore hypothesized that HDAC6 might promote cell migration and angiogenesis through interacting with EB1. To test this possibility, HUVECs were transfected with HDAC6 or EB1 siRNAs, together with pEGFPC1, pEGFPC1-HDAC6 or pEGFPC1-EB1. Cells were then scratched, and wound margins were examined $24 \mathrm{~h}$ later. As shown in Fig. 6A and 6B, HDAC6 siRNA-induced cell migration defect was largely rescued by GFP-HDAC6 and GFP-EB1. In contrast, EB1 siRNA-induced migration defect was rescued by GFP-EB1 but not GFP-HDAC6. These data suggest that EB1 is important for the regulation of endothelial cell migration by HDAC6. By endothelial tube formation and capillary sprouting assays, we further found that HDAC6 siRNA-induced in vitro angiogenesis defects could be rescued by both GFP-HDAC6 and GFP-EB1 (Fig. 6C-F). Taken together, these results indicate that HDAC6 promotes cell migration and angiogenesis in an EB1-dependent manner.

\section{DISCUSSION}

HDACs play important roles in diverse biological processes, primarily via their negative regulation of gene expression (Haberland et al., 2009). Most of the HDACs are localized in the nucleus and repress gene transcription through the highly conserved deacetylase domain. Studies using trichostain $A$, a pan-inhibitor of HDACs, have shown that HDACs are critically involved in angiogenesis (Kim et al., 2001). However, the contribution of individual HDACs to angiogenesis is largely unknown. In this study, we have analyzed the involvement of HDAC6, a cytoplasmic localized deacetylase, in the angiogenic process. Our data reveal that blocking HDAC6 activity by a specific inhibitor, tubacin, impairs angiogenesis both in chick embryos and in mice. In addition, we find that inhibition of HDAC6 activity or knockdown of its expression significantly diminishes endothelial tube formation and capillary sprouting in vitro. These results thus demonstrate a crucial role for HDAC6 in angiogenesis.

Previous studies have identified HDAC5 as a negative regulator of angiogenesis and $\mathrm{HDAC} 7$ as a positive regulator of angiogenesis (Chang et al., 2006; Urbich et al., 2009). Interestingly, both HDAC5 and HDAC7 undergo active nucleocytoplasmic shuttling in response to extracellular signals (McKinsey et al., 2000; Vega et al., 2004), which may allow for fine-tuning of angiogenesis-related genes. In contrast, HDAC6 resides exclusively in the cytoplasm presumably due to its tetradecapeptide repeat domain and its association with cytoskeletal components (Bertos et al., 2004; Valenzuela-Fernández et al., 2008). Consistent with the localization pattern of HDAC6, it mainly participates in cytoskeleton-related activities, independently of histone modification (Boyault et al., 2007; Valenzuela-Fernández et al., 2008). In this scenario, it is likely that the action of HDAC6 in angiogenesis is independent of gene transcription 
A

B
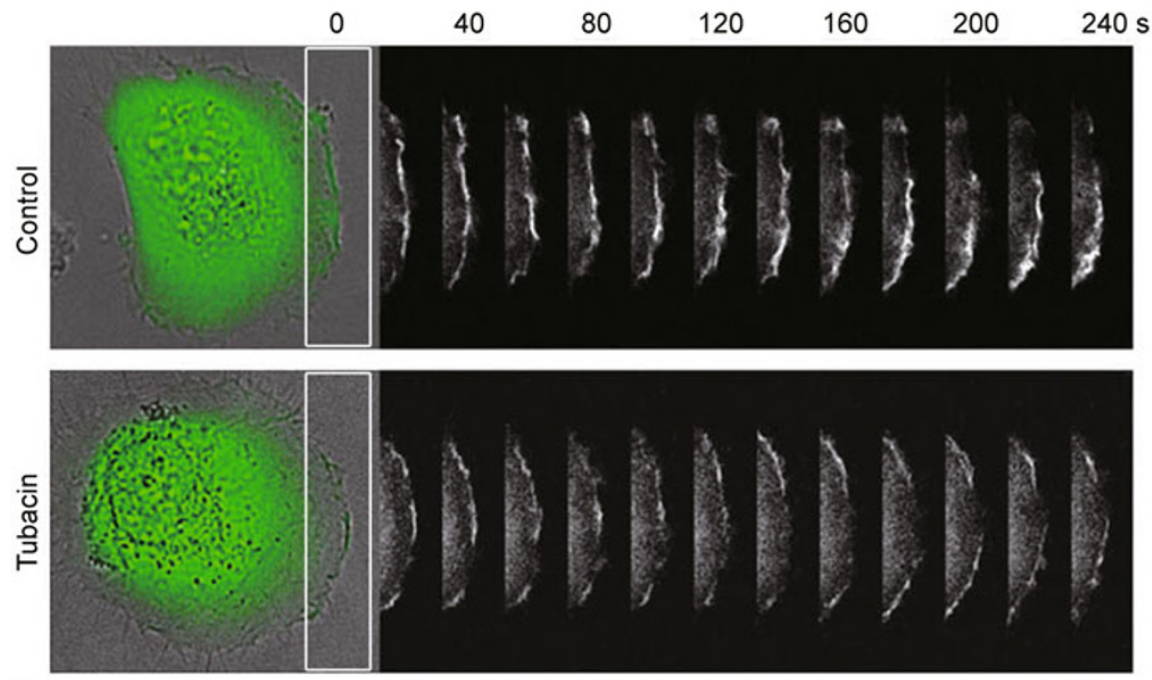

C
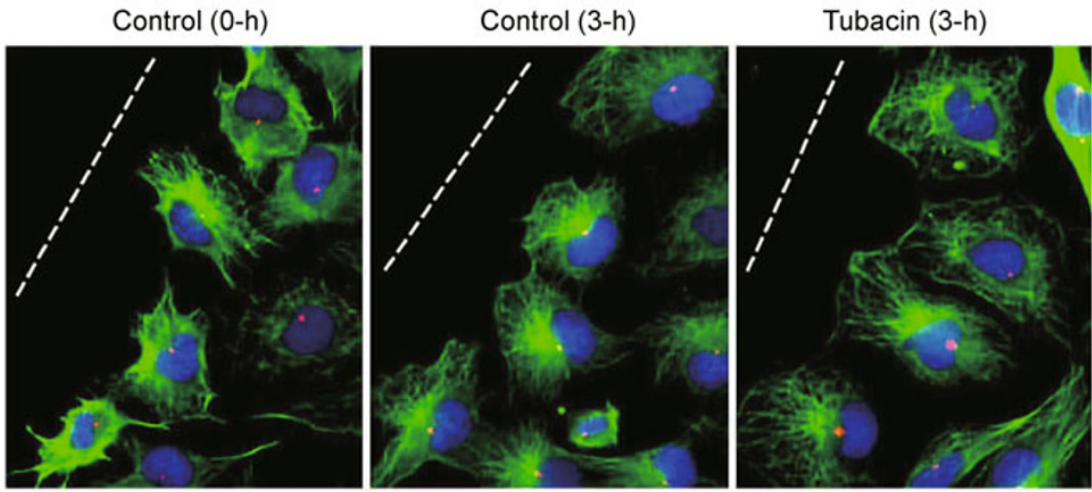

D

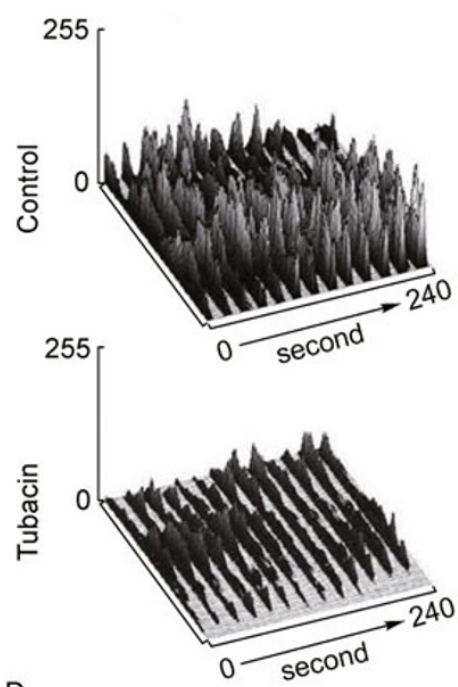

$\mathrm{E}$
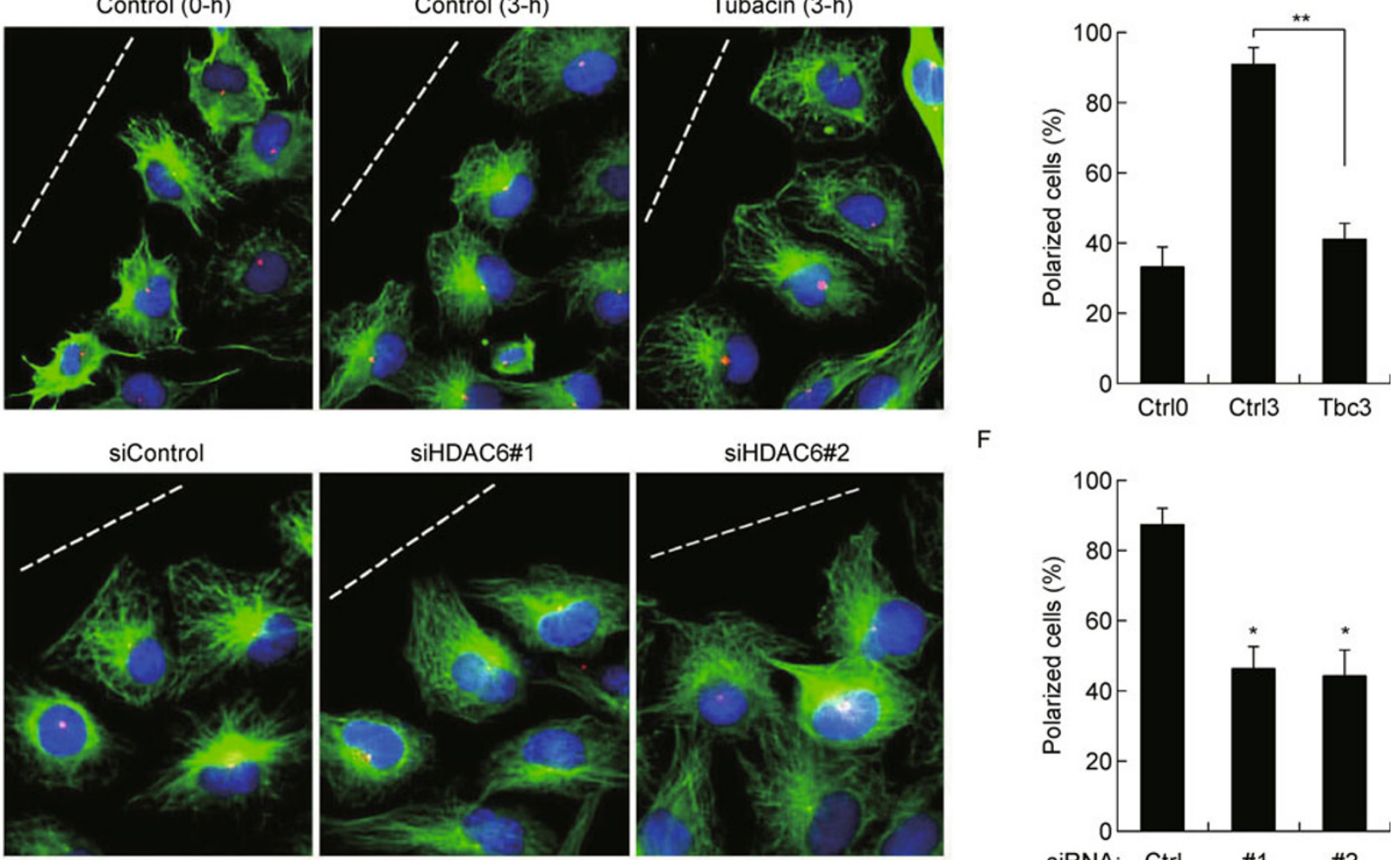

$\mathrm{F}$

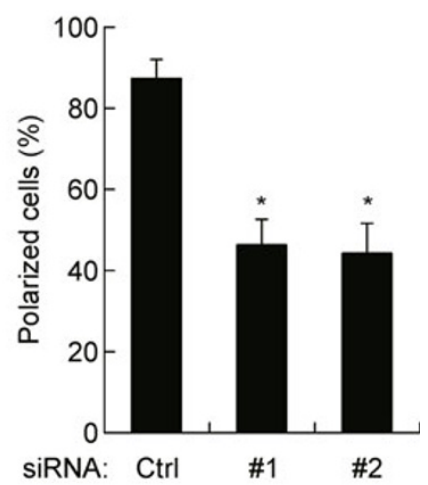

Figure 5. HDAC6 stimulates membrane ruffling and cell polarization. (A) HUVECs transfected with pEGFPC1 were treated with DMSO or tubacin and scratched. The fluorescence of GFP at the leading edge of cells was recorded at 20-second intervals. Rectangular regions were selected as indicated to analyze membrane ruffle dynamics. (B) Experiments were performed as in panel $A$, and membrane ruffle dynamics were presented as three-dimensional surface plots. (C) HUVECs were treated with DMSO or tubacin and scratched, and cells were fixed $3 \mathrm{~h}$ later and stained with anti- $\alpha$-tubulin antibody, anti-pericentrin antibody, and DAPI to visualize microtubules (green), centrosomes (red), and nuclei (blue), respectively. Broken white lines indicate the wound direction. (D) Experiments were performed as in panel $\mathrm{C}$, and the percentage of polarized cells at the wound margin was quantified (** $p<0.01)$. (E and F) HUVECs transfected with control or HDAC6 siRNAs were scratched, and the extent of cell polarization was examined as in panels $C$ and $D\left({ }^{*} p<0.05\right.$ vs control). 
A
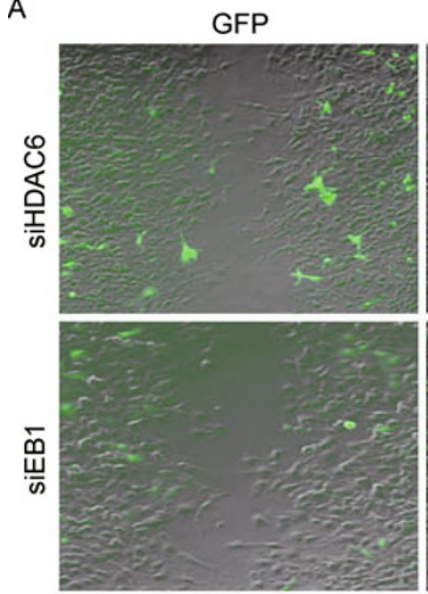

C
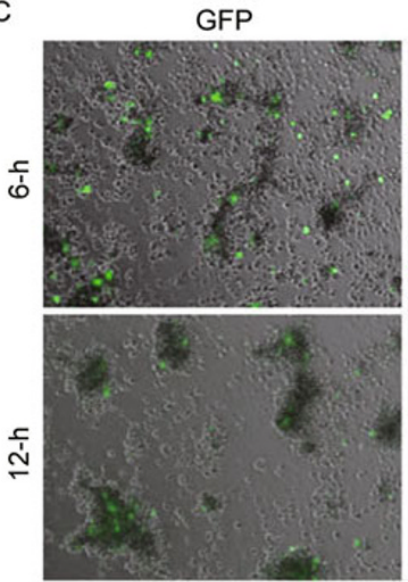

E

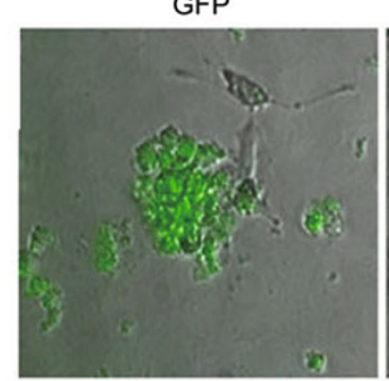

GFP-HDAC6
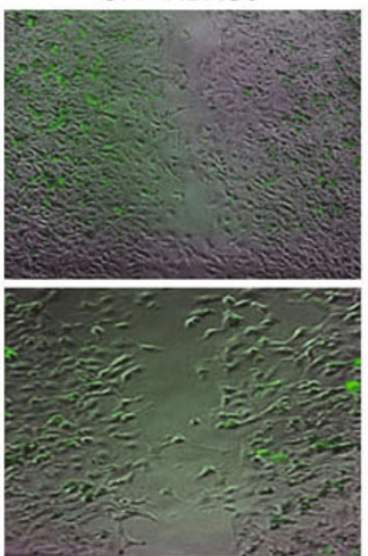

GFP-HDAC6
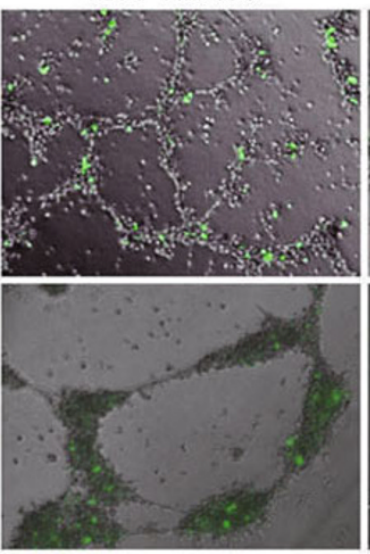

GFP-HDAC6

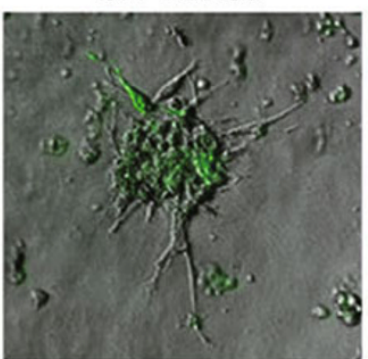

GFP-EB1
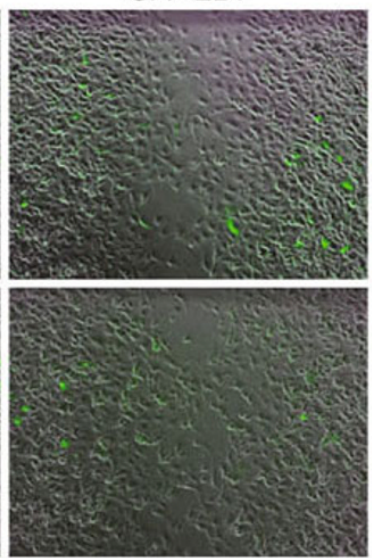

GFP-EB1

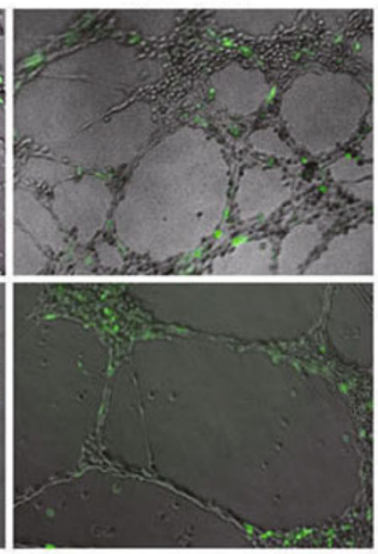

GFP-EB1

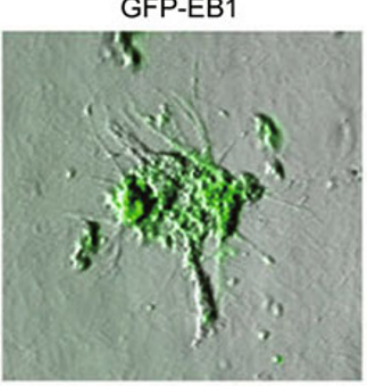

B

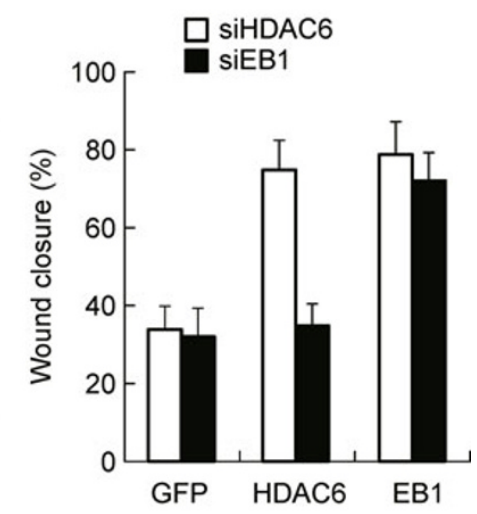

D

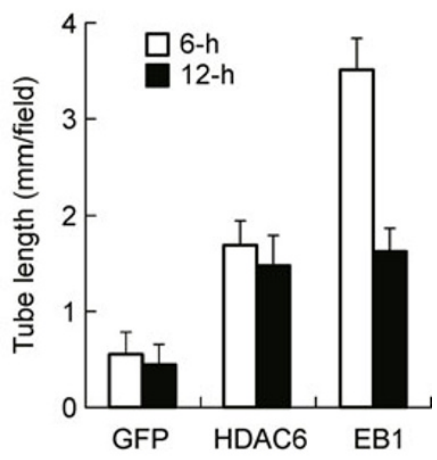

$\mathrm{F}$

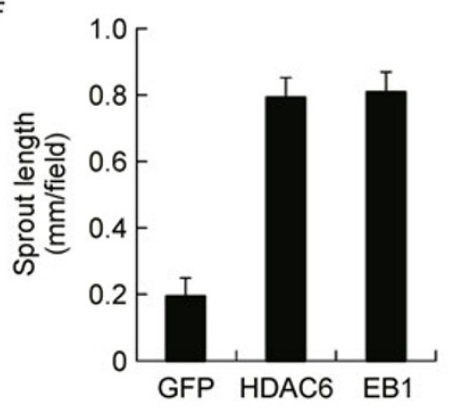

Figure 6. HDAC6 acts on EB1 to promote cell migration and angiogenesis. (A) HUVECs were transfected with HDAC6 or EB1 siRNAs, together with pEGFPC1, pEGFPC1-HDAC6 or pEGFPC1-EB1. Cells were scratched, and wound margins were examined with fluorescence microscopy $24 \mathrm{~h}$ later. (B) Experiments were performed as in panel $\mathrm{A}$, and the extent of wound closure was quantified. (C) HUVECs were transfected with HDAC6 siRNA together with pEGFPC1, pEGFPC1-HDAC6 or pEGFPC1-EB1, and the formation of endothelial tubes was examined with fluorescence microscopy 6 or $12 \mathrm{~h}$ later. (D) Experiments were performed as in panel $\mathrm{C}$, and cumulative tube length was quantified. (E) HUVECs were transfected with HDAC6 siRNA together with pEGFPC1, pEGFPC1-HDAC6 or pEGFPC1-EB1, and capillary-like sprout formation from the endothelial spheroids was examined with fluorescence microscopy. (F) Experiments were performed as in panel $\mathrm{E}$, and cumulative sprout length was quantified.

but instead involves cytoskeleton-associated events. This is supported by our finding that HDAC6 stimulates membrane ruffling at the leading edge of vascular endothelial cells and promotes cell polarization and migration, which are important steps in angiogenesis and rely on dynamic rearrangement of actin filaments and microtubules (Rodriguez et al., 2003; Lamalice et al., 2007).

Our data show that the microtubule-tip associated protein 
EB1 is important for HDAC6 to promote endothelial cell migration, providing further evidence that HDAC6 exerts its functions in endothelial cells through acting on the cytoskeleton. In agreement with this notion, overexpression of EB1 in vascular endothelial cells could largely rescue HDAC6 siRNA-induced defects in cell migration and formation of capillary/tube-like structures. Although the precise mechanism by which the HDAC6-EB1 interaction facilitates endothelial cell migration is unknown, it is possible that EB1 may help recruit HDAC6 to the cytoskeleton, thereby promoting the actions of HDAC6 in the vicinity of the cell cortex. Alternatively, the HDAC6-EB1 complex may serve as a scaffold for the binding of additional proteins important for cell migration.

Given our finding that HDAC6 is important for both embryonic and postnatal angiogenesis, it is surprising that HDAC6-deficient mice are viable and do not display obvious phenotype (Zhang et al., 2008). It is possible that HDAC10, another member of the HDAC family that is closely related to HDAC6 with functions less understood (Fischer et al., 2002; Guardiola and Yao, 2002), may complement the loss of HDAC6 in mice. In addition, it is conceivable that HDAC6 mainly functions in pathologic settings in mammals (e.g., during vascular injury or malignancy). Recent studies have demonstrated that HDAC6-deficient mice are resistant to carcinogen-induced skin tumors, suggesting a critical role for this deacetylase in cancer development (Lee et al., 2008). Therefore, it would be of great importance to investigate in the future the potential role of HDAC6 in tumor angiogenesis.

\section{MATERIALS AND METHODS}

\section{Cell culture and transfection}

Human umbilical vein endothelial cells (HUVECs) were cultured in RPMI 1640 medium supplemented with $10 \%$ fetal bovine serum at $37^{\circ} \mathrm{C}$ in a humidified atmosphere with $5 \% \quad \mathrm{CO}_{2}$. Plasmids were transfected into cells by electroporation with the ECM830 system (BTX), and small interfering RNAs (siRNAs) were transfected with the Lipofectamine 2000 reagent (Invitrogen).

\section{Endothelial cell migration, tube formation, and capillary sprouting}

HUVECs grown in 24-well plates as confluent monolayers were mechanically scratched using a pipette tip to create the wound. Cells were washed with phosphate-buffered saline to remove the debris, and complete culture medium was added to allow for wound healing. Phase contrast images of the wound were then taken at different time points. To examine the formation of tube-like structures, HUVECs were seeded onto 6-well plates precoated with matrigel, and tube formation was then examined at various time points. The extent of tube formation was quantified by measuring the cumulative tube length with ImageJ (National Institutes of Health). To examine capillary sprouting, HUVECs were suspended in culture medium containing $0.25 \%$ carboxymethylcellulose and seeded onto round- bottom 96-well plates to form spheroids. The spheroids were then embedded into collagen gels in 24-well plates, and culture medium was added on top of the gel. Capillary-like sprout formation was then examined by microscopy and angiogenic activity was quantified by measuring the cumulative sprout length per spheroid.

\section{Chick embryo angiogenesis assays}

Fertilized white $\mathrm{Hy}$-Line chicken eggs were incubated at $38^{\circ} \mathrm{C}$ in a humidified egg incubator. In one group of experiments, shells were opened at day 3 , and the whole embryos were transferred to $100-\mathrm{mm}$ petri dishes and cultured at $37^{\circ} \mathrm{C}$ in a standard humidified cell culture incubator. At day 4, filter paper soaked with tubacin or DMSO was placed on the vitelline membrane of the embryo, and capillaries underneath the filter paper were photographed 3 days later with an M165FC stereomicroscope (Leica). In another group of experiments, eggs were incubated in the egg incubator for 4 days, and a small window was then made by gently cracking the egg shell with forceps. The inner shell membrane was carefully removed without damaging the chorioallantoic membrane. At day 10, matrigel polymerized around two layers of nylon mesh, with DMSO or tubacin, was placed on the chorioallantoic membrane, and blood vessel growth into matrigel/nylon mesh was examined 3 days later.

\section{Directed angiogenesis in mice}

Angiogenic response in mice was examined as described previously (Gao et al., 2010). In brief, semiclosed angioreactors were filled with matrigel containing heparin, fibroblast growth factor 2 (FGF2), and tubacin or DMSO. The angioreactors were incubated at $37^{\circ} \mathrm{C}$ for $1 \mathrm{~h}$ to allow for gel formation and then implanted subcutaneously into the dorsal flank of 6- to 8-week-old athymic nude mice. After 11 days, the angioreactors were removed and photographed. Cell pellets were then recovered from the angioreactors by dispase digestion and stained with fluorescein-conjugated lectin-1 (FITC-lectin). The use of animals was approved by the Animal Care and Use Committee of Nankai University.

\section{Immunofluorescence microscopy}

HUVECs grown on glass coverslips were fixed with $4 \%$ paraformaldehyde and incubated with antibodies against $\alpha$-tubulin (SigmaAldrich) and pericentrin (Santa Cruz Biotechnology), and then with FITC- and rhodamine-conjugated secondary antibodies (Jackson ImmunoResearch Laboratories) followed by staining with 4',6diamidino-2-phenylindole (DAPI). Coverslips were mounted with $90 \%$ glycerol in phosphate-buffered saline and examined with an Axio Observer A1 fluorescence microscope (Carl Zeiss). For immunofluorescent staining of blood vessels in angioreactors, frozen sections of the vessel-containing matrigel were mounted onto slides precoated with 3-aminopropyltriethoxysilane. The sections were fixed with paraformaldehyde, stained with anti-CD31 antibody (Biolegend) and DAPI, and then examined by fluorescence microscopy. To examine HDAC6 localization in the vascular endothelium, frozen sections of mouse liver, small intestine, adrenal gland, and esophagus tissues were stained with antibodies against HDAC6 and CD31 and then examined by fluorescence microscopy. 


\section{Examination of membrane ruffling}

HUVECs transfected with pEGFPC1 were cultured in a $37^{\circ} \mathrm{C}$ chamber on a TCS SP5 confocal microscope (Leica), equipped with a live-cell imaging workstation. The fluorescence of GFP at the leading edge of cells was recorded at 20-second intervals using the LASAF software. The acquired image sequences were analyzed by ImageJ, and membrane ruffle dynamics were presented as threedimensional surface plots.

\section{ACKNOWLEDGEMENTS}

We thank Ruming Liu and Qiang Zhao for their technical assistance and members of the Zhou laboratory for helpful discussions. This work was supported by grants from the National Natural Science Foundation of China (Grant Nos. 30825022 and 90913021), the Fok Ying Tung Education Foundation (Grant No. 111036), and the National Basic Research Program of China (Grant No. 2007CB914802).

\section{ABBREVIATIONS}

EB1, microtubule end binding protein 1; FGF2, fibroblast growth factor 2; HDAC6, histone deacetylase 6; HUVECs, human umbilical vein endothelial cells; siRNAs, small interfering RNAs

\section{REFERENCES}

Bertos, N.R., Gilquin, B., Chan, G.K., Yen, T.J., Khochbin, S., and Yang, X.J. (2004). Role of the tetradecapeptide repeat domain of human histone deacetylase 6 in cytoplasmic retention. J Biol Chem 279, 48246-48254.

Bornens, M. (2008). Organelle positioning and cell polarity. Nat Rev Mol Cell Biol 9, 874-886.

Boyault, C., Sadoul, K., Pabion, M., and Khochbin, S. (2007). HDAC6, at the crossroads between cytoskeleton and cell signaling by acetylation and ubiquitination. Oncogene 26, 5468-5476.

Chang, S., Young, B.D., Li, S., Qi, X., Richardson, J.A., and Olson, E. N. (2006). Histone deacetylase 7 maintains vascular integrity by repressing matrix metalloproteinase 10 . Cell 126, 321-334.

Fischer, D.D., Cai, R., Bhatia, U., Asselbergs, F.A., Song, C., Terry, R., Trogani, N., Widmer, R., Atadja, P., and Cohen, D. (2002). Isolation and characterization of a novel class II histone deacetylase, HDAC10. J Biol Chem 277, 6656-6666.

Folkman, J. (2007). Angiogenesis: an organizing principle for drug discovery? Nat Rev Drug Discov 6, 273-286.

Gao, J., Sun, L., Huo, L., Liu, M., Li, D., and Zhou, J. (2010). CYLD regulates angiogenesis by mediating vascular endothelial cell migration. Blood 115, 4130-4137.

Guardiola, A.R., and Yao, T.P. (2002). Molecular cloning and characterization of a novel histone deacetylase HDAC10. J Biol Chem 277, 3350-3356.

Haberland, M., Montgomery, R.L., and Olson, E.N. (2009). The many roles of histone deacetylases in development and physiology: implications for disease and therapy. Nat Rev Genet 10, 3242.
Haggarty, S.J., Koeller, K.M., Wong, J.C., Grozinger, C.M., and Schreiber, S.L. (2003). Domain-selective small-molecule inhibitor of histone deacetylase 6 (HDAC6)-mediated tubulin deacetylation. Proc Natl Acad Sci U S A 100, 4389-4394.

Kim, M.S., Kwon, H.J., Lee, Y.M., Baek, J.H., Jang, J.E., Lee, S.W., Moon, E.J., Kim, H.S., Lee, S.K., Chung, H.Y., et al. (2001). Histone deacetylases induce angiogenesis by negative regulation of tumor suppressor genes. Nat Med 7, 437-443.

Lamalice, L., Le Boeuf, F., and Huot, J. (2007). Endothelial cell migration during angiogenesis. Circ Res 100, 782-794.

Lansbergen, G., and Akhmanova, A. (2006). Microtubule plus end: a hub of cellular activities. Traffic 7, 499-507.

Lee, Y.S., Lim, K.H., Guo, X., Kawaguchi, Y., Gao, Y., Barrientos, T., Ordentlich, P., Wang, X.F., Counter, C.M., and Yao, T.P. (2008). The cytoplasmic deacetylase HDAC6 is required for efficient oncogenic tumorigenesis. Cancer Res 68, 7561-7569.

Li, R., and Gundersen, G.G. (2008). Beyond polymer polarity: how the cytoskeleton builds a polarized cell. Nat Rev Mol Cell Biol 9, 860-873.

Marks, P.A. (2007). Discovery and development of SAHA as an anticancer agent. Oncogene 26, 1351-1356.

McKinsey, T.A., Zhang, C.L., Lu, J., and Olson, E.N. (2000). Signaldependent nuclear export of a histone deacetylase regulates muscle differentiation. Nature 408, 106-111.

Rodriguez, O.C., Schaefer, A.W., Mandato, C.A., Forscher, P., Bement, W.M., and Waterman-Storer, C.M. (2003). Conserved microtubule-actin interactions in cell movement and morphogenesis. Nat Cell Biol 5, 599-609.

Semenza, G.L. (2007). Vasculogenesis, angiogenesis, and arteriogenesis: mechanisms of blood vessel formation and remodeling. J Cell Biochem 102, 840-847.

Urbich, C., Rössig, L., Kaluza, D., Potente, M., Boeckel, J.N., Knau, A., Diehl, F., Geng, J.G., Hofmann, W.K., Zeiher, A.M., et al. (2009). HDAC5 is a repressor of angiogenesis and determines the angiogenic gene expression pattern of endothelial cells. Blood 113, 5669-5679.

Valenzuela-Fernández, A., Cabrero, J.R., Serrador, J.M., and Sánchez-Madrid, F. (2008). HDAC6: a key regulator of cytoskeleton, cell migration and cell-cell interactions. Trends Cell Biol 18, 291-297.

Vega, R.B., Harrison, B.C., Meadows, E., Roberts, C.R., Papst, P.J., Olson, E.N., and McKinsey, T.A. (2004). Protein kinases C and D mediate agonist-dependent cardiac hypertrophy through nuclear export of histone deacetylase 5. Mol Cell Biol 24, 8374-8385.

Xu, W.S., Parmigiani, R.B., and Marks, P.A. (2007). Histone deacetylase inhibitors: molecular mechanisms of action. Oncogene 26, 5541-5552.

Zhang, Y., Kwon, S., Yamaguchi, T., Cubizolles, F., Rousseaux, S., Kneissel, M., Cao, C., Li, N., Cheng, H.L., Chua, K., et al. (2008). Mice lacking histone deacetylase 6 have hyperacetylated tubulin but are viable and develop normally. Mol Cell Biol 28, 1688-1701.

Zilberman, Y., Ballestrem, C., Carramusa, L., Mazitschek, R., Khochbin, S., and Bershadsky, A. (2009). Regulation of microtubule dynamics by inhibition of the tubulin deacetylase HDAC6. J Cell Sci 122, 3531-3541. 